\title{
Cambios esqueléticos cefalométricos en la base craneal post expansión rápida del maxilar
}

\author{
Cephalometric skeletal changes on the cranial base post rapid \\ maxillary expansion.
}

\section{Resumen}

La Expansión Rápida del Maxilar (ERM) ha sido clásicamente descrita en la literatura como un procedimiento terapéutico indicado en el tratamiento de deficiencia transversa maxilar. El objetivo del presente estudio fue evaluar cefalométricamente los cambios esqueléticos a nivel de la base craneal en pacientes en crecimiento sometidos a expansión rápida del maxilar. El material estuvo constituido por radiografías cefalométricas tomadas de 10 pacientes en crecimiento (ocho de sexo masculino y dos de sexo femenino), con necesidad de expansión rápida del maxilar como parte de su tratamiento ortodóncico. El rango de edad de los pacientes fue de 7.1 a 12.7 años, y la edad media fue de $10.6 \pm 2.04$ años. Se obtuvieron radiografías cefalométricas laterales de cada paciente antes del tratamiento $\left(\mathrm{T}_{1}\right)$ e inmediatamente después de la fase activa de tratamiento $\left(\mathrm{T}_{2}\right)$. Las variables evaluadas fueron: ángulo NSBa, ángulo NBa/F.H., ángulo SN/ENA-ENP, ángulo F.H./ENA-ENP, ángulo SN/F.H., distancia S-N, distancia $\mathrm{S}-\mathrm{Ba}$, distancia N-Ba. Los datos se analizaron estadísticamente utilizando la prueba t de Student para muestras pareadas $(\mathrm{p}<0.05)$. De acuerdo con el análisis estadístico, se encontraron cambios significativos en todas las variables evaluadas. Por lo tanto, se puede especular que la expansión rápida del maxilar no sólo genera efectos esqueléticos locales sino también cambios esqueléticos en la base craneal.

Palabras Clave: Expansión Rápida del Maxilar; Base craneal; Cefalometría.

\section{Abstract}

Rapid Maxillary Expansion (ERM) has been classically described in the literature as a therapeutic procedure in the treatment of transverse maxillary deficiency. The aim of this study was to evaluate the cephalometric skeletal changes on the cranial base in growing patients who had undergone rapid maxillary expansion.

The material consisted of lateral radiographs taken from ten growing patients (eight male and two female) with need of rapid maxillary expansion as part of their orthodontic treatment. The age range of the patients was 7.1 to 12.7 years, and the mean age was $10.6 \pm 2.04$ years. Lateral radiographs of each patient were taken before treatment $\left(\mathrm{T}_{1}\right)$ and immediately after the active phase of treatment $\left(\mathrm{T}_{2}\right)$. The evaluated variables were: NSBa angle, NBa/F.H. angle, SN/ENA-ENP angle, F.H./ENA-ENP angle, SN/F.H. angle, S-N distance, S-Ba distance and N-Ba distance. The data were statistically analyzed using the paired Student's t-test $(\mathrm{p}<0.05)$. According to the statistical analysis, significant changes were found in all the evaluated variables. Therefore, it can be speculated that rapid maxillary expansion has had not only local skeletal effects but also significant skeletal changes on the cranial base.

Keywords: Rapid maxillary expansion; Cranial base; Cephalometry.

\section{Introducción}

La expansión rápida del maxilar (ERM) fue introducida en el siglo XIX y ha sido clásicamente descrita en la literatura como un procedimiento terapéutico indicado en el tratamiento de deficiencia transversa maxilar. La ERM se produce cuando las fuerzas transversales aplicadas a los dientes y procesos maxilares superan el límite necesario para el movimiento ortodóncico dental. La presión aplicada actúa como una fuerza ortopédica que produce la apertura gradual de la sutura palatina media separando los huesos maxilares ${ }^{1}$. Aunque el principal efecto de la expansión rápida del maxilar se concentra en esta zona, no se limita a ella sino que parece implicar a una amplia porción del complejo cráneofacial, que incluyen estructuras directa o indirectamente relacionados con el maxilar como la mandíbula ${ }^{1,2}$, cavidad nasal ${ }^{1,2}$, suturas cráneo-faciales y circunmaxilares ${ }^{4-10}$ e incluso estructuras anatómicas profundas del cráneo como las apófisis pterigoides del hueso esfenoides ${ }^{4,5}$.

La inquietud por conocer los efectos de la expansión rápida del maxilar sobre la base craneal ha sido motivo de múltiples investigaciones basadas en métodos histológicos ${ }^{4}$, gammagrafía ósea $^{10}$, tomografía computarizada ${ }^{11} y$ modelos de elementos finitos (FEM) ${ }^{5-}$ 9,12 .

\section{Artículo Original}

\section{Beatriz Chileno-Aquiño ${ }^{1}$, Manuel Chávez-Sevillano ${ }^{2}$}

1. Escuela Académico Profesional de Odontología de la Universidad Nacional Mayor de San Marcos.

2. Departamento Académico de Estomatología Pediátrica de la Facultad de Odontología de la Universidad Nacional Mayor de San Marcos. Perú.

\section{Correspondencia:}

C.D. Beatriz Chileno-Aquiño

Urb. Las Fresas Mz. R Lt. 8, Callao, Perú

Correo electrónico: chileno.beatriz@gmail.com

Coautor:

Chavez-Sevillano: gustavochs@gmail.com

Fecha de recepción: 27-10-14

Fecha de aceptación: 04-12-14
Un estudio histológico en monos Rhesus $^{4,14}$ y dos estudios en humanos ${ }^{10,11,13}$ demostraron apertura de la sincondrosis esfeno-occipital y aumento significativo de la actividad metabólica en el área de la sincondrosis esfeno-occipital tras la aplicación de expansión rápida del maxilar.

Con respecto a los estudios FEM, a pesar de sus limitaciones, la mayoría de las investigaciones ${ }^{6-9,12}$ excepto una ${ }^{5}$ revelaron aumento en los niveles de estrés en la base del cráneo (especialmente en las partes superiores de las placas pterigoides). Holberg y cols. ${ }^{7,8}$. concluyeron que el estrés inducido por la expansión rápida del maxilar conlleva a moderadas tensiones y deformaciones 
en la base craneal de niños y adolescentes. Gautam y cols. ${ }^{9}$ observaron que el área correspondiente a la sincondrosis esfeno-occipital sufrió desplazamiento en dirección antero-inferior, por lo que sugirieron que la sincondrosis esfenooccipital podría sufrir remodelación ósea en respuesta a la expansión rápida del maxilar lo que apoya las conclusiones de Gardner \& Kronman ${ }^{4}$ y Ba$\operatorname{ydas}^{10}$.

Es por ello, que el propósito de la presente investigación fue evaluar cefalométricamente los cambios esqueléticos que ocurren a nivel de la base craneal en pacientes en crecimiento post expansión rápida del maxilar.

\section{Materiales y método}

El material del presente estudio prospectivo estuvo constituido por radio-

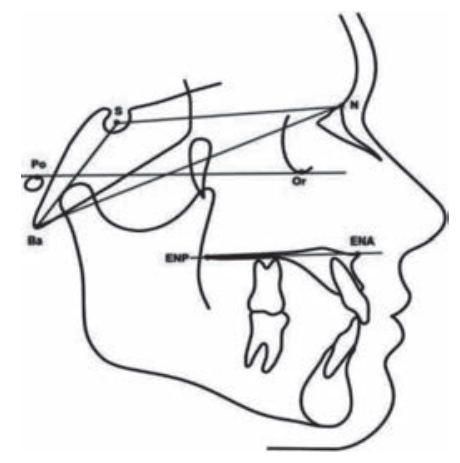

Fig. 1 Esquema del trazado cefalométrico.

\section{Análisis Estadístico. Error de Método}

Para evaluar los posibles errores operacionales intra-examinador, todas las mediciones se realizaron dos veces con un intervalo de 30 días por el mismo examinador calibrado.

Se utilizó la fórmula de Dahlberg y la prueba t-Student para muestras pareadas, con un nivel de significancia del 5\% para evaluar el error casual y sistemático respectivamente. El error casual varió entre 0.15 y 0.27 en la fase $T_{1}$ y entre 0.11 y 0.19 en la fase $\mathrm{T}_{2}$. Ninguna variable analizada mostró error sistemático estadísticamente significativo en ninguna fase.

La estadística descriptiva (media, desviación estándar), fue calculada por separado para $\mathrm{T}_{1} \mathrm{y} \mathrm{T}_{2}$. La prueba de Shapiro-Wilk, $(\mathrm{p}<0.05)$ determinó distribución normal de los datos. Las medias de los datos en $\mathrm{T}_{1}$ y $\mathrm{T}_{2}$ se compararon utilizando la prueba t-Student para muestras pareadas con un nivel de significancia de 5\%. Los grafías cefalométricas tomadas de diez pacientes en crecimiento (ocho de sexo masculino y dos de sexo femenino) con deficiencia maxilar transversal tratados con expansión rápida del maxilar en la Clínica del Niño y Adolescente de la Facultad de Odontología de la Universidad Nacional Mayor de San Marcos. La edad promedio fue de 10,6 \pm 2.04 años (rango: 7,1 a 12,7 ańos). Pacientes con enfermedad inflamatoria aguda o alteración congénita/hereditaria que comprometiera el desarrollo maxilar o aquellos con tratamiento ortodóncico u ortopédico previo no fueron incluidos en este estudio.

Los pacientes fueron tratados con expansores tipo Hass y tipo Hyrax, los cuales fueron activados con dos vuel- tas $(0,25 \mathrm{~mm} /$ vuelta $)$ en el momento de instalación y los días siguientes a razón de dos vueltas/ día, una por la mañana y una por la noche intercalando dos días activos consecutivos por uno de descanso durante un tiempo promedio de 21 días, tiempo en que se evidenció la apertura de la sutura palatina.

A cada paciente se le tomaron radiografías cefalométricas laterales antes de la expansión rápida del maxilar $\left(\mathrm{T}_{1}\right)$ e inmediatamente al final de la fase activa de expansión $\left(\mathrm{T}_{2}\right)$ sin remover el expansor. Sobre cada radiografía se trazaron de forma manual las estructuras anátomo-radiológicas y se ubicaron los puntos cefalométricos de interés para la evaluación de las siguientes ocho variables: (Fig. 1).

(1) ^ NSBa; Ángulo Násion-Sella-Básion, Angulo de la Base craneal

(2) ^ NBa/F.H: Ángulo Násion-Básion/Plano Horizontal de Frankfort, Ángulo de deflexión craneal

(3) ^ SN/F.H: Ángulo Sella-Násion/Plano Horizontal de Frankfort

$(4) \wedge$ SN/ENA-ENP: Ángulo Sella-Násion/ENA-ENP

(5) ^ F.H/ENA-ENP: Ángulo Plano Horizontal de Frankfort/ENA-ENP

(6) Distancia S-N: Distancia Sella-Násion, base craneal anterior.

(7) Distancia S-Ba: Distancia Sella-Básion, base craneal posterior.

(8) Distancia N-Ba: Distancia Násion-Básion, base craneal total.

datos fueron analizados utilizando el paquete estadístico SPSS versión 20.0 para Windows (SPSS Inc., Chicago, Illinois, USA).

\section{Resultados}

La estadística descriptiva para las mediciones de las variables en la fase pre ERM (T1) y post fase activa de ERM (T2) y para los cambios como resultado de ERM (T2-T1) se presentan en la Tablas No 1, 2 y 3.

Tabla 1. Análisis descriptivo de las variables cefalométricas pre ERM (T1)

\begin{tabular}{lcccc}
\hline Variable & Media & D.E & Máx. & Mín. \\
\hline$\wedge \mathrm{NSBa}\left({ }^{\circ}\right)$ & 130.1 & 2.904 & 133.5 & 125.5 \\
$\wedge$ NBa/F.H. $\left({ }^{\circ}\right)$ & 27.9 & 11,738 & 30 & 26 \\
$\wedge$ SN/F.H $\left(^{\circ}\right)$ & 8.7 & 1.7826 & 11 & 6 \\
$\wedge$ SN/ENA-ENP $\left(^{\circ}\right)$ & 7.45 & 2.4883 & 11 & 3.5 \\
$\wedge$ F.H/ENA-ENP $\left({ }^{\circ}\right)$ & -26 & 2.5413 & 4.5 & -4.5 \\
distancia S-N $(\mathrm{mm})$ & 70.4 & 2.6437 & 75 & 65.5 \\
distancia S-Ba $(\mathrm{mm})$ & 45.85 & 2.0419 & 48 & 42 \\
distancia N-Ba $(\mathrm{mm})$ & 106.2 & 2.9833 & 112 & 102 \\
\hline
\end{tabular}

Tabla 2. Análisis descriptivo de las variables cefalométricas post ERM (T2)

\begin{tabular}{lllll}
\hline \multicolumn{1}{c}{ Variable } & Media & D.E & Máx. & Mín. \\
\hline$\wedge$ NSBa $\left({ }^{\circ}\right)$ & 130.8 & 2.9926 & 134 & 126 \\
$\wedge$ NBa/F.H. $\left({ }^{\circ}\right)$ & 27.1 & 1.3499 & 29.5 & 25 \\
$\wedge$ SN/F.H. $\left({ }^{\circ}\right)$ & 8.3 & 1.9178 & 11 & 5.5 \\
$\wedge$ SN/ENA-ENP $\left({ }^{\circ}\right)$ & 8.6 & 2.675 & 12.5 & 5 \\
$\wedge$ F.H/ENA-ENP $\left({ }^{\circ}\right)$ & 0.3 & 2.8206 & 6.5 & -3 \\
distancia S-N $(\mathrm{mm})$ & 71 & 2.5604 & 75.5 & 66.5 \\
distancia S-Ba $(\mathrm{mm})$ & 46.5 & 2.1344 & 49 & 42.5 \\
distancia N-Ba $(\mathrm{mm})$ & 107.15 & 2.9064 & 112.5 & 103 \\
\hline
\end{tabular}

Los resultados de la prueba t-Student para muestras pareadas se presentan en la Tabla 3. Todas las variables examinadas mostraron cambio significativo entre los periodos T1 y T2 ( $<<0,001)$. Se registró aumento significativo post fase activa de expansión rápida del maxilar en las dimensiones angulares $\mathrm{NSBa}\left(0.70^{\circ} \pm 0.2582\right)$, SN/ENA$\operatorname{ENP}\left(1.15^{\circ} \pm 0.5297\right)$ y F.H./ENA$\operatorname{ENP}\left(1.55^{\circ} \pm 0.4972\right)$, así como en las dimensiones lineales Sella-Násion $(0.60 \mathrm{~mm} \pm 0.3162)$, Sella-Básion 
$(0.65 \mathrm{~mm} \pm 0.2415)$ y Násion-Básion $(0.95 \mathrm{~mm} \pm 0.2838)$. Mientras que los ángulos $\mathrm{NBa} / \mathrm{F} . \mathrm{H}$. y SN/F.H experimentaron disminución significativa de $0.80^{\circ} \pm 0.2582$ y $0.40^{\circ} \pm 0.3162$ respectivamente.

Tabla 3. Análisis comparativo de las variables cefalométricas como resultado de la ERM mediante la prueba t-Student para muestras pareadas

\begin{tabular}{|c|c|c|c|c|}
\hline \multirow{2}{*}{ Variable } & \multicolumn{2}{|c|}{ Cambios (T2-T1) } & \multirow{2}{*}{$\mathrm{t}$} & \multirow{2}{*}{$\mathbf{P}$} \\
\hline & Media & D.E & & \\
\hline$\wedge \mathrm{NSBa}\left({ }^{\circ}\right)$ & 0.7 & 0.2582 & 8.573 & $<0.001^{*}$ \\
\hline$\wedge \mathrm{NBa} / \mathrm{F} . \mathrm{H} .\left(^{\circ}\right)$ & 0.8 & 0.2582 & 9.798 & $<0.001^{*}$ \\
\hline$\wedge$ SN/F.H $\left({ }^{\circ}\right)$ & 0.4 & 0.3162 & 4 & $<0.001^{*}$ \\
\hline$\wedge$ SN/ENA-ENP( $\left(^{\circ}\right)$ & 1.15 & 0.5297 & 6.866 & $<0.001^{*}$ \\
\hline$\wedge$ F.H/ENA-ENP $\left({ }^{\circ}\right)$ & 1.55 & 0.4972 & 9.858 & $<0.001^{*}$ \\
\hline S-N (mm) & 0.6 & 0.3162 & 6 & $<0.001^{*}$ \\
\hline S-Ba (mm) & 0.65 & 0.2415 & 8.51 & $<0.001^{*}$ \\
\hline $\mathrm{N}-\mathrm{Ba}(\mathrm{mm})$ & 0.95 & 0.2838 & 10.585 & $<0.001^{*}$ \\
\hline
\end{tabular}

T1, Mediciones pre ERM; T2, mediciones post ERM; $\left({ }^{*}\right)$ diferencia estadísticamente significativa

\section{Discusión}

Con respecto al ángulo NSBa, el aumento estadísticamente significativo podría estar en relación directa con hallazgos previos de apertura y desplazamiento de la sincondrosis esfeno-occipital durante el proceso de expansión rápida del maxilar. Gardner y Kronman ${ }^{4}$ mediante un estudio histológico en monos Rhesus, evidenciaron separación de los segmentos óseos alrededor de la sincondrosis esfeno-occipital, con un desplazamiento de 0,5 a $1 \mathrm{~mm}$. Gautam y cols. ${ }^{9}$ valiéndose de un modelo de elementos finitos (FEM) observaron desplazamiento antero-inferior de la sincondrosis esfeno-occipital, lo que demostraba remodelación ósea en respuesta a expansión rápida del maxilar. Así también, Leonardi y cols. ${ }^{11}$ mediante un estudio tomográfico evidenciaron ligera apertura de la sincondrosis esfeno occipital en pacientes jóvenes. Sato ${ }^{15}$ relaciona las modificaciones en el área de la sincondrosis esfeno-occipital con cambios en la angulación de la base craneal y resalta la importancia de ésta sobre el compleo craneofacial.

Por otro lado, el presente resultado no coincide con los hallazgos de Jafari y cols. ${ }^{5}$ quienes reportaron permanencia constante del ángulo de la base craneal y nulo desplazamiento de la sincondrosis esfeno-occipital post expansión rápida del maxilar respectivamente.

En relación al ángulo $\mathrm{NBa/F.H.,} \mathrm{la}$ disminución estadísticamente significativa, indicaría una posible inclinación horaria de la base craneal $(\mathrm{N}$ Ba) con respecto a F.H., sin embargo más pareciera estar asociada al movi- miento posterior de Básion causado por la apertura del ángulo NSBa que a un descenso de Násion, tal y como afirmaron Courtney y cols. ${ }^{16}$ quienes asociaron el desplazamiento posterior de $0.86 \mathrm{~mm}$ de Básion con una apertura del ángulo NSBa de $0.44^{\circ}$ en pacientes tratados con aparatos funcionales. Esto parece confirmarse más aún, al considerar que pese a que el ángulo SN/F.H, disminuye de manera estadísticamente significativa, la variable no cambió en la totalidad de pacientes, permaneciendo constante en algunos de ellos, lo que también cuestionaría la rotación horaria de la base craneal anterior por descenso del punto Násion.

Desde este punto de vista, los aumentos estadísticamente significativos en los ángulos SN/ENA-ENP y FH/ ENA-ENP, estarían relacionados al descenso y rotación horaria del maxilar más que a un cambio de orientación de las líneas representativas de la base craneal (línea Sella-Násion y plano Horizontal de Frankfort) concordando con lo afirmado por Haas, ${ }^{1}$ Da Silva y cols. ${ }^{2}$ y Garib y cols. ${ }^{3}$

Con respecto a las dimensiones lineales, los aumentos estadísticamente significativos post expansión rápida del maxilar de las variables S-N, S-Ba y $\mathrm{N}-\mathrm{Ba}$ estarían relacionados de manera directa con la apertura del ángulo NSBa, que posicionaría el punto Básion posteriormente, aumentando la distancia S-Ba y a su vez de la distancia N-Ba. Esto concuerda con los estudios de Sato, ${ }^{15}$ y Singh y cols. ${ }^{17}$ en los que hubo aumento de la longitud S-N como en el ángulo NSBa y los estudios de Sato ${ }^{15}$ y Dhopatkar y cols. ${ }^{18}$ en los que hubo aumento de la longitud S-Ba al mismo tiempo que apertura del ángulo NSBa.

En relación al aumento del ángulo de la base craneal (NSBa) y disminución del ángulo de deflexión craneal $\mathrm{NBa} / \mathrm{F} . \mathrm{H}$ podríamos inferir que la expansión rápida del maxilar provoca un efecto favorable en pacientes con atresia maxilar, sin embargo podría resultar contraproducente en pacientes con retrognatismo mandibular y plano mandibular empinado, pues empeoraría la relación sagital máxilomandibular a la vez que pronunciaría el patrón facial convexo característico de este tipo de maloclusión, tal y como ha sido advertido anteriormente por Haas ${ }^{1}$ quien aconsejaba especial cautela en pacientes con plano mandibular empinado, altura facial antero inferior aumentada, perfil convexo o patrón de crecimiento dólicofacial.

\section{Conclusiones}

Se encontraron cambios estadísticamente significativos en todas las variables evaluadas como son: ángulo $\mathrm{NSBa}$, ángulo NBa/F.H., ángulo SN/ ENA-ENP, ángulo F.H./ENA-ENP, ángulo $\mathrm{SN} / \mathrm{F} . \mathrm{H}$., distancia $\mathrm{S}-\mathrm{N}$, distancia S-Ba y distancia N-Ba. Aunque los cambios encontrados son en promedio menores que $2 \mathrm{~mm}$, lo cual podría ser atribuido al error del método, se puede afirmar que la expansión rápida del maxilar no sólo genera efectos esqueléticos locales sino también cambios esqueléticos en la base craneal, región donde se localiza la sincondrosis esfeno-occipital.

Cabe destacar que estos hallazgos aunque son estadísticamente significativos podrían tener poca significan- 
cia clínica dada la pequeña magnitud de cambios.

\section{Referencias bibliográficas}

1. Haas AJ. Longterm posttreatment evaluation of rapid palatal expansion. Angle Orthod. 1980;50:189-217.

2. Da Silva Filho OG, Villas Boas MC, Capelozza Filho L. Rapid maxillary expansion in the primary and mixed dentitions: A cephalometric evaluation. Am J Orthod Dentofacial Orthop 1991;100(2): 171-181

3. Garib DG, Henriques JF, Carvalho PE, Gomes SC. Longitudinal effects of rapid maxillary expansion. Angle Orthod. 2007; $77(3): 442-8$

4. Gardner GE, Kronmann JH. Cranioskeletal displacement caused by rapid palatal expansion in the rhesus monkey. Am J Orthod. 1971;59:146-155.

5. Jafari A, Shetty KS, Kumar M. Study of stress distribution and displacement of various craniofacial structures following application of transverse orthopedic forcesa three-dimensional FEM study. Angle Orthod. 2003;73:12-20.

6. ISeri H, Tekkaya AE, O* ztan O* , Bilgic, S. Biomechanical effect of rapid maxillary expansion on the craniofacial skeleton, studied by the finite element method. Eur J Orthod.1998;20:347-356

7. Holberg C. Effects of rapid maxillary expansion on the cranial base-an FEM-analysis. J Orofac Orthop. 2005;66:54- 66.

8. Holberg C, Rudzki-Janson I. Stresses at the cranial base induced by rapid maxillary expansion. Angle Orthod. 2006; 76:543-550.

9. Gautam P, Valiathan A, Adhikari R. Stress and displacement patterns in the craniofacial skeleton with rapid maxillary expansion: a finite element method study. Am J Orthod Dentofacial Orthop 2007;132:5.e1-511.

10. Baydas B, Yavuz I, Uslu H, Dagsuyu IM, Ceylan I. Nonsurgical rapid maxillary expansion effects on craniofacial structures in young adult females: a bone scintigraphy study. Angle Orthod. 2006;76:759-767.

11. Leonardi R, Cutrera A, Barbato E. Rapid maxillary expansion affects the spheno-occipital synchondrosis in youngsters. A study with low-dose computed tomography. Angle Orthod. 2010;80:106-110

12. Boryor A, Geiger M, Hohmann A, Wunderlich A, Sander C, Martin Sander F, et al. Stress distribution and displacement analysis during an intermaxillary disjunction-a three-dimensional FEM study of a human skull. J Biomech. 2008; 41:376-382.

13. Lieberman DE, Pearson OM, Mowbray KM. Basicranial influence on overall cranial shape. J Human Evolut 2000; 38: 291-315

14. Lieberman DE, Ross CF, Ravosa MJ. The primate cranial base: ontogeny, function, and integration. Am J Phys Anthropol 2000;Suppl 31:117-169.

15. Sato S.A. Treatment Approach to Malocclusion Under the Consideration of Craniofacial Dynamics. Grace Printing Press INC, Phillipines, 2001.

16. Courtney M, Harkness M, Herbison P. Maxillary and cranial base changes during treatment with functional appliances. Am J Orthod Dentofacial Orthop. 1996;109:616-24.

17. Singh GD, McNamara JA, Lozanoff S. Finite element analysis of the cranial base in subjects with class III malocclusion. Br J Orthod. 1997; 24:103-112.

18. Dhopatkar A, Bhatia S, Rock P. An investigation into the relationship between the cranial base angle and malocclusion. Angle Orthod 2002; 72: 456-63. 